\title{
Revisiting well-posed boundary conditions for the shallow water equations
}

\author{
Sarmad Ghader and Jan Nordström
}

\section{Linköping University Post Print}

\section{Tweet}

N.B.: When citing this work, cite the original article.

Original Publication:

Sarmad Ghader and Jan Nordström, Revisiting well-posed boundary conditions for the shallow water equations, 2014, Dynamics of atmospheres and oceans (Print), (66), 1-9.

http://dx.doi.org/10.1016/j.dynatmoce.2014.01.002

Copyright: Elsevier

http://www.elsevier.com/

Postprint available at: Linköping University Electronic Press

http://urn.kb.se/resolve?urn=urn:nbn:se:liu:diva-103645 


\title{
Revisiting well-posed boundary conditions for the shallow water equations
}

\author{
Sarmad Ghader* \\ Institute of Geophysics, University of Tehran, Tehran, Iran \\ Jan Nordström \\ Division of Computational Mathematics, Department of Mathematics, Linköping University, SE-581 83 Linköping, \\ Sweden
}

\begin{abstract}
We derive a general form of well-posed open boundary conditions for the two-dimensional shallow water equations by using the energy method. Both the number and the type of boundary conditions are presented for subcritical and supercritical flows on a general domain. The boundary conditions are also discussed for a rectangular domain. We compare the results with a number of often used open boundary conditions and show that they are a subset of the derived general form.
\end{abstract}

Keywords: Shallow water equations, Well-posedness, Boundary conditions, Energy method, Subcritical, Supercritical

\section{Introduction}

The single layer shallow water models are extensively used in numerical studies of large scale atmospheric and oceanic motions. This model describes a fluid layer of constant density in which the horizontal scale of the flow is much greater than the layer depth. The dynamics of the single layer model is less general than three dimensional models, but is often preferred because of its mathematical and computational simplicity (Pedlosky (1987); Vallis (2006)).

Well-posed boundary conditions are an essential requirement for all stable numerical schemes developed for initial boundary value problems. For any limited area atmospheric or oceanic numerical model the lateral boundaries of the domain are not physical boundaries and artificial boundary conditions are used. The boundary conditions used at these artificial boundaries must lead to a well-posed problem. In addition, the boundary conditions must generate stable solutions for the discretized version of the governing equations. Furthermore, the boundaries should be as transparent as possible. The focus of the present work is on the first crucial step in this chain, namely the derivation of a general form of the well-posed boundary conditions for the two-dimensional shallow water equations and the other issues are not considered here.

\footnotetext{
${ }^{*}$ Corresponding author.

Email addresses: sghader@ut .ac.ir (Sarmad Ghader), jan.nordstrom@liu.se (Jan Nordström)
} 
For the one-dimensional shallow water equations, well-posed boundary conditions have been derived previously by transforming them into a set of decoupled scalar equations (Durran (2010)). Oliger and Sundström (1978) derived well-posed boundary conditions for several sets of partial differential equations including the shallow water equations by using the energy method. We will follow the path set by Oliger and Sundström (1978) and derive a general form of well-posed open boundary conditions for the two-dimensional shallow water equations. The core mathematical tool is the energy method where one bounds the energy of the solution by choosing a minimal number of suitable boundary conditions (Gustafsson et al. (1995); Nordström and Svärd (2005); Gustafsson (2008)).

Secondly, it is shown that the open boundary conditions proposed by others such as Oliger and Sundström (1978), Mcdonald (2002) and Blayo and Debreu (2005) are special cases of the new general form of derived open boundary conditions in this paper.

The remainder of this paper is organized as follows. The shallow water equations are given in section 2. Section 3 gives the various definitions of well-posedness. In sections 4 and 5 , well-posedness of the shallow water equations and well-posed boundary conditions for the two dimensional shallow water equations for a general domain are derived. The boundary conditions for a rectangular domain, as an example, are also presented. The details of the proposed open boundary conditions and the relation to work of others are presented in section 6 . Finally, concluding remarks are given in section 7 .

\section{The shallow water equations}

The inviscid single-layer shallow water equations, including the Coriolis term, are (Vallis (2006))

$$
\begin{aligned}
\frac{D \mathbf{V}}{D t}+f \hat{\mathbf{k}} \times \mathbf{V}+g \nabla h & =0 \\
\frac{D h}{D t}+h \nabla \cdot \mathbf{V} & =0
\end{aligned}
$$

where $\mathbf{V}=u \hat{\mathbf{i}}+v \hat{\mathbf{j}}$ is the horizontal velocity vector with $u$ and $v$ being the velocity components in $x$ and $y$ directions, respectively. $\hat{\mathbf{i}}$ and $\hat{\mathbf{j}}$ are the unit vectors in $x$ and $y$ directions, respectively. $h$ represents the surface height, $D() / D t=\partial() / \partial t+(\mathbf{v} \cdot \nabla)()$ is the substantial time derivative, $f$ is the Coriolis parameter and $g$ is the acceleration due to gravity. The unit vector in vertical direction is denoted by $\hat{\mathbf{k}}$. Here, we use the $f$-plane approximation where the Coriolis parameter is taken to be a constant.

The vector form of the two-dimensional shallow water equations, linearized around a constant basic state, can be written as

$$
\mathbf{u}_{t}+\mathbf{A} \mathbf{u}_{x}+\mathbf{B} \mathbf{u}_{y}+\mathbf{C u}=0
$$

where the subscripts $t, x$ and $y$ denote the derivatives. The definition of the vector $\mathbf{u}$ and the matrices $\mathbf{A}, \mathbf{B}$ and $\mathbf{C}$ is

$$
\mathbf{u}=\left(\begin{array}{c}
u^{\prime} \\
v^{\prime} \\
h^{\prime}
\end{array}\right), \mathbf{A}=\left(\begin{array}{ccc}
U & 0 & g \\
0 & U & 0 \\
H & 0 & U
\end{array}\right), \mathbf{B}=\left(\begin{array}{lll}
V & 0 & 0 \\
0 & V & g \\
0 & H & V
\end{array}\right), \mathbf{C}=\left(\begin{array}{ccc}
0 & -f & 0 \\
f & 0 & 0 \\
0 & 0 & 0
\end{array}\right) .
$$

Here, $u^{\prime}$ and $v^{\prime}$ are the perturbation velocity components and $h^{\prime}$ is the perturbation height. In addition, $U, V$ and $H$ represent the constant mean fluid velocity components and height. 


\section{Well-posedness}

Before embarking on the derivation of well-posed boundary conditions of the shallow water equations, we need to define well-posedness. An initial boundary value problem, such as the two-dimensional shallow water equations in a limited area domain, is said to be well-posed if there exists a unique solution that depends continuously on the initial and boundary data.

To define the well-posedness in a mathematical sense, consider the following initial boundary value problem

$$
\begin{aligned}
\frac{\partial \mathbf{q}}{\partial t} & =\mathbf{P q}+\mathbf{F}, \quad \mathbf{x} \in \Omega, \quad t \geq 0 \\
\mathbf{L q} & =\mathbf{g}, \quad \mathbf{x} \in \partial \Omega, \quad t \geq 0 \\
\mathbf{q} & =\mathbf{f}, \quad \mathbf{x} \in \Omega, \quad t=0
\end{aligned}
$$

where $\mathbf{q}$ is the solution, $\mathbf{x}$ is the space vector, $\mathbf{P}$ is a spatial differential operator and $\mathbf{L}$ is the boundary operator. $\mathbf{F}$ is a forcing function, $\mathbf{g}$ and $\mathbf{f}$ are boundary and initial functions, respectively. $\mathbf{F}, \mathbf{g}$ and $\mathbf{f}$ are the known data of the problem. In our analysis it is assumed that $\mathbf{P}$ and $\mathbf{L}$ are linear operators.

We need the following definition.

Definition 1. Consider the problem (4). The differential operator $\mathbf{P}$ is called semi-bounded if for all $\mathbf{q} \in \mathbb{V}$, where $\mathbb{V}$ being the space of differentiable functions satisfying the boundary conditions $\mathbf{L q}=0$, the inequality

$$
(\mathbf{q}, \mathbf{P q}) \leq \alpha\|\mathbf{q}\|^{2}
$$

holds. In (5), $\alpha$ is a constant independent of q. Here, ( , ) and \|\| denote the scalar product and norm, respectively.

The estimate (5) guarantees that an energy estimate exists for (4). However, to many boundary conditions could have been used, which means that no solution exist. To guarantee existence, we also need the following definition.

Definition 2. The differential operator $\mathbf{P}$ is maximally semi-bounded if it is semi-bounded in the function space $\mathbb{V}$ but not semi-bounded in any space with fewer boundary conditions.

Finally, the following theorem relates maximal semi-boundedness and well-posedness.

Theorem 1. Consider the initial boundary value problem (4). If the operator $\mathbf{P}$ is maximally semi-bounded, then the initial boundary value problem with $\mathbf{g}=0$ is well-posed.

More details on these definitions, theorem and proofs are given by Gustafsson et al. (1995) and Gustafsson (2008).

As will be shown below, we will follow the path set by others and perform the analysis on the linearized constant coefficient problem. This is no limitation since by using the linearization and localization principles (Kreiss and Lorenz, 1989) it can be shown that if the constant coefficient and linearized form of an initial boundary value system is well-posed then the associated original nonlinear problem is also well-posed. The linearization principle express that if the linear problems which are obtained by linearizing at all functions near $\mathbf{q}$ (solution) are well-posed, then the associate nonlinear problem is well-posed at $\mathbf{q}$. The localization principle states that if all frozen coefficient problems are well-posed then the associate variable-coefficient problem is also well-posed. For more details, see the "linearization and localization" principles in Kreiss and Lorenz (1989) as well as in Strang (1964). 


\section{Well-posedness of the shallow water equations}

The linearized constant coefficient two-dimensional shallow water equations (3) with initial and boundary conditions can be formulated as

$$
\begin{aligned}
\mathbf{u}_{t}+\mathbf{A} \mathbf{u}_{x}+\mathbf{B} \mathbf{u}_{y}+\mathbf{C u} & =0 \quad(x, y) \in \Omega, \quad t \geq 0 \\
L \mathbf{u}(x, y, t) & =\mathbf{g}(x, y, t), \quad(x, y) \in \partial \Omega, \quad t \geq 0 \\
\mathbf{u}(x, y, 0) & =\mathbf{f}(x, y), \quad(x, y) \in \Omega, \quad t=0
\end{aligned}
$$

where $\mathbf{f}$ is the initial data and $\mathbf{g}$ the boundary data. $L$ is the boundary operator, and will be the main focus in this paper. It can be seen that the spatial operator $\mathbf{P}$ of equation (4), for the system of equations (6) is $\mathbf{P}=-\mathbf{A} \mathbf{u}_{x}-\mathbf{B} \mathbf{u}_{y}-\mathbf{C u}$.

To be able to integrate by parts we need to symmetrize the equations (Abarbanel and Gottlieb, 1981; Nordström and Svärd, 2005). Therefore, equation (6) is rewritten as:

$$
(\mathbf{S u})_{t}+\mathbf{S A S}^{-1}(\mathbf{S u})_{x}+\mathbf{S B S}^{-1}(\mathbf{S u})_{y}+\mathbf{S C S}^{-1}(\mathbf{S u})=0 \text {. }
$$

The symmetrizing matrix $\mathbf{S}$ used in (9), is

$$
\mathbf{S}=\left(\begin{array}{ccc}
1 & 0 & 0 \\
0 & 1 & 0 \\
0 & 0 & \frac{g}{c}
\end{array}\right)
$$

where $c=\sqrt{g H}$ is the gravity wave speed. The matrices $\mathbf{A}^{s}=\mathbf{S} \mathbf{A} \mathbf{S}^{-1}$ and $\mathbf{B}^{s}=\mathbf{S B S}^{-1}$ must be symmetric and we find that

$$
\mathbf{A}^{s}=\left(\begin{array}{ccc}
U & 0 & c \\
0 & U & 0 \\
c & 0 & U
\end{array}\right), \quad \mathbf{B}^{s}=\left(\begin{array}{ccc}
V & 0 & 0 \\
0 & V & c \\
0 & c & V
\end{array}\right)
$$

where $\mathbf{C}^{s}=\mathbf{S C S}^{-1}=\mathbf{C}$. The new variable which transforms equation (6) to a symmetric form is found as

$$
\mathbf{v}=\mathbf{S u}=\left(u^{\prime}, v^{\prime}, g h^{\prime} / c\right)^{T},
$$

where the superscript $T$ denotes transpose. Therefore, equation (6) is transformed to

$$
\mathbf{v}_{t}+\mathbf{A}^{s} \mathbf{v}_{x}+\mathbf{B}^{s} \mathbf{v}_{y}+\mathbf{C v}=0
$$

The usual Euclidean $L_{2}$ scalar product and norm that we will use are

$$
(\mathbf{u}, \mathbf{v})=\int_{\Omega} \mathbf{u v} d x d y, \quad\|\mathbf{u}\|^{2}=(\mathbf{u}, \mathbf{u}) .
$$

Next we need to compute (u, Pu) [see Definition 1 and equation (5)]. To this end, multiplying equation (10) by $\mathbf{v}^{T}$ followed by integration over the domain leads to

$$
\|\mathbf{v}\|_{t}^{2}+\int_{\Omega}\left(\mathbf{v}^{T} \mathbf{A}^{s} \mathbf{v}\right)_{x} d x d y+\int_{\Omega}\left(\mathbf{v}^{T} \mathbf{B}^{s} \mathbf{v}\right)_{y} d x d y=0 .
$$


The term containing $\mathbf{C}$, due to its skew-symmetry, is zero. ${ }^{1}$ Using Gauss' theorem on equation (11) we find

$$
\|\mathbf{v}\|_{t}^{2}+\oint_{\partial \Omega}\left(\mathbf{v}^{T} \hat{\mathbf{A}} \mathbf{v}\right) d s=0 .
$$

In (12), $\hat{\mathbf{A}}=\hat{\mathbf{n}} \cdot\left(\mathbf{A}^{s}, \mathbf{B}^{s}\right), \hat{\mathbf{n}}=\left(n_{x}, n_{y}\right)=(d y,-d x) / d s$ is the outward pointing unit vector on the surface $\partial \Omega$ and $d s=\sqrt{d x^{2}+d y^{2}}$.

After finding the eigenvalues of $\hat{\mathbf{A}}$, the right eigenvectors can be used to write

$$
\mathbf{\Lambda}=\mathbf{R}^{T} \mathbf{A} \mathbf{R}
$$

where

$$
\mathbf{R}=\left(\begin{array}{ccc}
-\frac{n_{x}}{\sqrt{2}} & -n_{y} & \frac{n_{x}}{\sqrt{2}} \\
-\frac{n_{y}}{\sqrt{2}} & n_{x} & \frac{n_{y}}{\sqrt{2}} \\
\frac{1}{\sqrt{2}} & 0 & \frac{1}{\sqrt{2}}
\end{array}\right), \quad \boldsymbol{\Lambda}=\left(\begin{array}{ccc}
\omega-c & 0 & 0 \\
0 & \omega & 0 \\
0 & 0 & \omega+c
\end{array}\right) .
$$

In (14), $\omega=n_{x} U+n_{y} V=(U, V) \cdot \hat{\mathbf{n}}$. Using the new variable $\mathbf{w}=\mathbf{R}^{T} \mathbf{v}$, equation (12) is rewritten as

$$
\|\mathbf{v}\|_{t}^{2}+\oint_{\partial \Omega} \mathbf{w}^{T} \boldsymbol{\Lambda} \mathbf{w} d s=0
$$

If the surface integral in equation (15) is positive then condition (5) will be satisfied. This implies that the spatial operator $\mathbf{P}=-\mathbf{A} \mathbf{u}_{x}-\mathbf{B} \mathbf{u}_{y}-\mathbf{C u}$ is semi-bounded, and based on Theorem 1, the problem will be well-posed. Therefore, equation (15) implies that the linearized two-dimensional shallow water equations will be well-posed if the surface integral in (15) is positive. In addition, $\|\mathbf{v}\|^{2}$ will decrease as a function of time which implies that it is bounded. Consequently, condition (15) can be used to find well-posed boundary conditions.

\section{Well-posed boundary conditions for a general domain}

The sign of $\omega=(U, V) \cdot \hat{\mathbf{n}}$ determines whether we have inflow or outflow. In other words, $\omega<0$ signals inflow and $\omega>0$ outflow. In addition we have

$$
\mathbf{w}^{T} \boldsymbol{\Lambda} \mathbf{w}=w_{1}^{2}(\omega-c)+w_{2}^{2} \omega+w_{3}^{2}(\omega+c)
$$

where

$$
w_{1}=\frac{\sqrt{2}}{2}\left(\frac{g h^{\prime}}{c}-\left(u^{\prime}, v^{\prime}\right) \cdot \hat{\mathbf{n}}\right), \quad w_{2}=-\left(u^{\prime}, v^{\prime}\right) \times \hat{\mathbf{n}}, \quad w_{3}=\frac{\sqrt{2}}{2}\left(\frac{g h^{\prime}}{c}+\left(u^{\prime}, v^{\prime}\right) \cdot \hat{\mathbf{n}}\right)
$$

To find well-posed boundary conditions for a general domain, condition (15) implies that

$$
\mathbf{w}^{T} \boldsymbol{\Lambda} \mathbf{w} \geq 0
$$

\footnotetext{
${ }^{1}$ It can be shown that the matrix $\mathbf{C}$ will be also skew-symmetric when the Coriolis parameter is not constant (in practical applications it is usually a function of latitude). Therefore, the analysis will be the same for this case.
} 
is necessary at both inflow and outflow boundaries. In addition, it should be noted that based on Definition 2 and Theorem 1 (see section 3), for well-posedness we need to satisfy condition (17) with a minimal number of boundary conditions.

Note that in order to obtain an energy-estimate and finally well-posedness, only Dirichlet type of boundary conditions are allowed. Condition (17) will become indefinite if mixed boundary conditions of Robin type are used, and Neumann boundary conditions are impossible since gradients are not present in (17).

For subcritical inflow where $|\omega|<c$, the components of the diagonal matrix $\boldsymbol{\Lambda}$ are $\omega<0$, $\omega-c<0$ and $\omega+c>0$. At a subcritical outflow boundary the components of the diagonal matrix $\boldsymbol{\Lambda}$ are $\omega>0, \omega-c<0$ and $\omega+c>0$. For the subcritical case, at the inflow boundary we have two negative eigenvalues. Therefore, to satisfy condition (17) we need two boundary conditions at the inflow boundary. At the outflow boundary there is only one negative eigenvalue and to satisfy condition (17), one boundary conditions is needed at the outflow boundary. This choice makes the spatial operator maximally semi-bounded, and well-posedness follows (see Section 3).

For supercritical inflow where $|\omega|>c$, the components of the diagonal matrix $\boldsymbol{\Lambda}$ are $\omega<0$, $\omega-c<0$ and $\omega+c<0$. At the outflow boundary, the components of the diagonal matrix $\boldsymbol{\Lambda}$ are $\omega>0, \omega-c>0$ and $\omega+c>0$. For this case, it is seen that at the inflow boundary we have three negative eigenvalues. Then, to satisfy condition (17) we need three boundary conditions at an inflow boundary. For the outflow boundary, all of the eigenvalues are positive and condition (17) is automatically satisfied. Therefore, we need no boundary conditions at an outflow boundary.

\subsection{Subcritical inflow and outflow boundaries}

The general form of two boundary conditions for the inflow case that will assure all terms in condition (17) are positive have the form

$$
w_{2}-\alpha_{3} w_{3}=0, \quad w_{1}-\beta_{3} w_{3}=0
$$

The form of (18) is due to the fact that $\omega+c$ is the only positive eigenvalue. By substituting (18) into (17), we arrive to the following relation

$$
\mathbf{w}^{T} \boldsymbol{\Lambda} \mathbf{w}=w_{3}^{2}\left[\beta_{3}^{2}(\omega-c)+\alpha_{3}^{2} \omega+\omega+c\right] \geq 0
$$

and then we find that the coefficients $\alpha_{3}$ and $\beta_{3}$ must satisfy

$$
\beta_{3}^{2}-\alpha_{3}^{2} \frac{\omega}{c-\omega} \leq \frac{c+\omega}{c-\omega}
$$

For a subcritical outflow boundary only one boundary condition is needed in order to satisfy condition (17). To assure that all terms are positive we can use the following general boundary condition

$$
w_{1}-\alpha_{2} w_{2}-\alpha_{4} w_{3}=0
$$

Again, the form of (19) is due to the fact that $\omega$ and $\omega+c$ are positive. By substituting (19) into (17) we have

$$
\mathbf{w}^{T} \boldsymbol{\Lambda} \mathbf{w}=w_{2}^{2}\left(\alpha_{2}^{2} \omega-\alpha_{2}^{2} c+\omega\right)+w_{3}^{2}\left(\alpha_{4}^{2} \omega-\alpha_{4}^{2} c+\omega+c\right)+2 \alpha_{2} \alpha_{4} \geq 0 .
$$

The relation (20) can be rewritten in the following matrix form

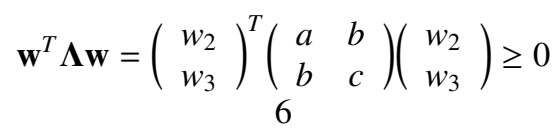


where

$$
a=\alpha_{2}^{2} \omega-\alpha_{2}^{2} c+\omega, \quad b=\alpha_{2} \alpha_{4}(\omega-c), \quad c=\alpha_{4}^{2} \omega-\alpha_{4}^{2} c+\omega+c .
$$

To satisfy condition (21), eigenvalues of matrix must be positive semi-definite, i.e.,

$$
\alpha_{2}^{2}+\alpha_{4}^{2} \frac{\omega}{c+\omega} \leq \frac{\omega}{c-\omega}
$$

\subsection{Supercritical inflow and outflow boundaries}

For the supercritical inflow boundary we need three boundary conditions to satisfy condition (17). They are

$$
w_{1}=w_{2}=w_{3}=0
$$

or any three linear combination of (22). Condition (17) is automatically satisfied at a supercritical outflow boundary and therefore no boundary conditions are needed in that case.

\subsection{The boundary conditions for a rectangular domain}

As an example, the general well-posed boundary conditions derived above are considered for a rectangular domain, which can be considered as a representative geometry used in real world two dimensional limited area models, see Figure 1.

It is assumed that the basic state velocity components are positive $(U>0, V>0)$. By this assumption, it can be seen that the west $\left(x=0,0 \leq y \leq L_{y}\right)$ and south $\left(y=0,0 \leq x \leq L_{x}\right)$ boundaries are of inflow type since $\omega<0$. The east $\left(x=L_{x}, 0 \leq y \leq L_{y}\right)$ and north $\left(y=L_{y}, 0 \leq\right.$ $x \leq L_{x}$ ) boundaries are of outflow type since $\omega>0$. Note that the crucial parameter which decides whether the flow is subcritical or supercritical is $|\omega|$, not the magnitude of the vector $(U, V)$.

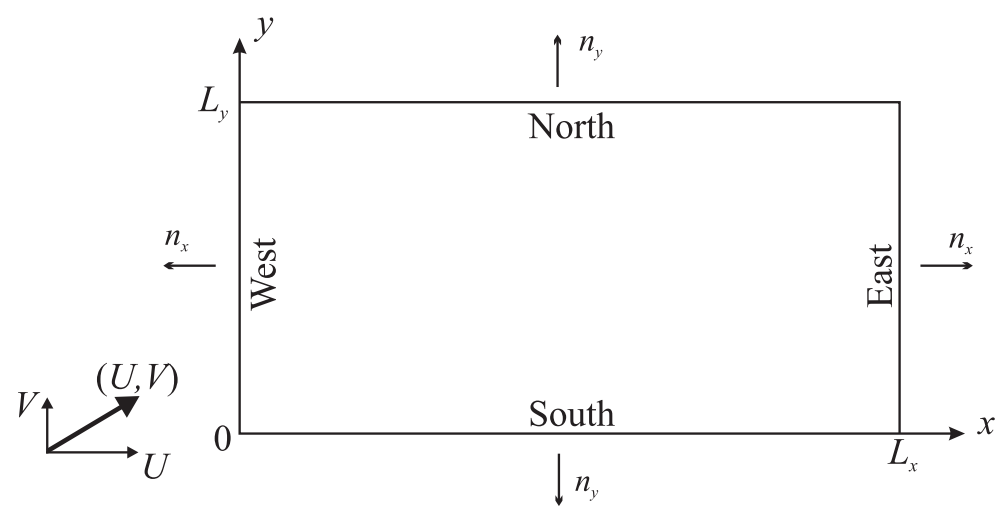

Figure 1: Rectangular domain

The summary of inflow and outflow boundary conditions for the subcritical and supercritical cases are given in Table 1. The table presents the number and type of the boundary conditions for the rectangular domain. 
Table 1: The number and type of boundary conditions for the rectangular domain.

\begin{tabular}{|c|c|c|}
\hline \multicolumn{3}{|c|}{ Subcritical Flow Case } \\
\hline Boundary & Number & Boundary Condition(s) \\
\hline South (inflow) & 2 & $\begin{array}{r}u^{\prime}-\alpha_{3} \frac{\sqrt{2}}{2}\left(\frac{g h^{\prime}}{c}-v^{\prime}\right)=0 \\
\frac{g h^{\prime}}{c}+v^{\prime}-\beta_{3}\left(\frac{g h^{\prime}}{c}-v^{\prime}\right)=0\end{array}$ \\
\hline West (inflow) & 2 & $\begin{aligned} v^{\prime}-\alpha_{3} \frac{\sqrt{2}}{2}\left(\frac{g h^{\prime}}{c}-u^{\prime}\right) & =0 \\
\frac{g h^{\prime}}{c}+u^{\prime}-\beta_{3}\left(\frac{g h^{\prime}}{c}-u^{\prime}\right) & =0\end{aligned}$ \\
\hline East (outflow) & 1 & $\frac{\sqrt{2}}{2}\left(\frac{g h^{\prime}}{c}-u^{\prime}\right)-\alpha_{2} v^{\prime}-\alpha_{4} \frac{\sqrt{2}}{2}\left(\frac{g h^{\prime}}{c}+u^{\prime}\right)=0$ \\
\hline North (outflow) & 1 & $\frac{\sqrt{2}}{2}\left(\frac{g h^{\prime}}{c}-v^{\prime}\right)+\alpha_{2} u^{\prime}-\alpha_{4} \frac{\sqrt{2}}{2}\left(\frac{g h^{\prime}}{c}+v^{\prime}\right)=0$ \\
\hline \multicolumn{3}{|c|}{ Supercritical Flow Case } \\
\hline Boundary & Number & Boundary Condition(s) \\
\hline Inflow & 3 & $u^{\prime}=v^{\prime}=h^{\prime}=0$ \\
\hline Outflow & None & None \\
\hline
\end{tabular}

\section{The relation to other open boundary conditions}

The homogeneous boundary conditions derived above will be used for the nonlinear shallow water equations at open boundaries by augmenting them with known data at boundaries.

The subcritical inflow open boundary conditions (Browning and Kreiss, 1982; Blayo and Debreu, 2005) can be written as

$$
\mathbf{Q}_{i} \mathbf{w}=\mathbf{Q}_{i} \mathbf{w}_{\mathrm{e}}
$$

where

$$
\mathbf{Q}_{i}=\left(\begin{array}{ccc}
0 & 1 & -\alpha_{3} \\
1 & 0 & -\beta_{3}
\end{array}\right), \quad \mathbf{w}=\left(w_{1}, w_{2}, w_{3}\right)^{T}
$$

In a similar way the subcritical outflow open boundary condition can be proposed as

$$
\mathbf{Q}_{o} \mathbf{w}=\mathbf{Q}_{o} \mathbf{w}_{\mathrm{e}}
$$

where

$$
\mathbf{Q}_{o}=\left(1,-\alpha_{2},-\alpha_{4}\right)
$$

In equations (23) and (24), the subscript e denotes known data at the boundary.

In fact, the open boundary conditions presented in equations (23) and (24) are more general than other boundary conditions presently proposed by other researches. Blayo and Debreu (2005) have shown that the classical open boundary conditions used in atmospheric and oceanic models can be revisited from the characteristic variables point of view. On the other hand, the characteristic variables are a subset of the derived general well-posed boundary conditions in the present work. Therefore, it is possible to revisit the mentioned atmospheric and oceanic open boundary conditions using the derived general well-posed boundary conditions.

If we choose $\alpha_{3}=0$ and $\beta_{1}= \pm 1$ in (23) we arrive at the special cases suggested by Oliger and Sundström (1978). In addition, if we choose $\alpha_{2}=0$ in (24) the coefficient $\alpha_{4}$ satisfies

$$
\left|\alpha_{4}\right| \leq \sqrt{8}_{\frac{c+\omega}{c-\omega}}
$$


and since $\left|\alpha_{4}\right| \geq 1$, the special choices

$$
w_{1}-w_{3}=0, \quad \text { or } \quad w_{1}+w_{3}=0
$$

are valid boundary conditions. The boundary condition (19) is more general but the specific boundary conditions (25) are less complex and easier to implement. The boundary conditions (25) expressed in primitive variables are $\left(u^{\prime}, v^{\prime}\right) \cdot \hat{\mathbf{n}}=0$ and $h^{\prime}=0$, respectively. In addition, it can be seen that the special cases presented in (25) are similar to that proposed by Oliger and Sundström (1978) by setting $\alpha_{2}=0$ and $\alpha_{4}= \pm 1$.

If we choose $\beta_{3}=0$ in (23) we arrive to the following boundary condition

$$
w_{1}=w_{1 \mathrm{e}}
$$

which is the same boundary condition proposed by Flather (1976) and Mcdonald (2002). If in (23) we choose $\beta_{3}=0$ and $\alpha_{3}=0$ we find

$$
\begin{aligned}
& w_{1}=w_{1 \mathrm{e}} \\
& w_{2}=w_{2 \mathrm{e}}
\end{aligned}
$$

at inflow boundary. In addition, if we choose $\alpha_{2}=\alpha_{4}=0$ in (24) we reach to the following equation

$$
w_{1}=w_{1 \mathrm{e}}
$$

at outflow boundary. It can be seen that the boundary conditions given in equations (27)-(29) are similar to boundary conditions derived by Blayo and Debreu (2005) for the shallow water equations.

\section{Concluding remarks}

We have derived well-posed boundary conditions for the two-dimensional shallow water equations by using the energy method. Well-posed boundary conditions including the type and number of boundary conditions have been derived for subcritical and supercritical inflow and outflow boundaries on a general two dimensional domain.

For the subcritical inflow case it was shown that we need two boundary conditions to bound the energy of the solution and at the subcritical outflow boundary only one boundary condition is needed. For the supercritical inflow case it was shown that three boundary conditions are required and at the outflow boundary none. The exact form of the boundary operator was determined for all four cases. In addition, as an example, a rectangular domain was considered and the specific well-posed boundary conditions at the inflow and outflow boundaries were extracted.

As the second aim of this work, we use the derived general form of the boundary conditions and it is shown that all other boundary conditions are found by using specific parameter choices. In particular, it was shown that some well-known open boundary conditions proposed by others are special cases of the newly derived open boundary conditions.

The next stage of the present work is to implement the derived well-posed boundary conditions in a numerical algorithm. High order finite difference approximations on summation by parts form with weak boundary procedures to implement the different types of boundary conditions derived in this paper will lead to energy stable approximations. 


\section{Acknowledgments}

The authors would like to thank universities of Tehran and Linköping for supporting this research work. We also thank the anonymous reviewers for their helpful comments on the manuscript.

\section{References}

Abarbanel, S., Gottlieb, D., 1981. Optimal time splitting for two- and three-dimensional Navier-Stokes equations with mixed derivatives. Journal of Computational Physics 41, 1-33.

Blayo, E., Debreu, L., 2005. Revisiting open boundary conditions from the point of view of characteristic variables Ocean Modelling 9, 231-252.

Browning, G.L., Kreiss, H.O., 1982. Initialization of the shallow water equations with open boundaries by the bounded derivative method. Tellus 34, 334-351.

Durran, D.R., 2010. Numerical methods for fluid dynamics with applications to geophysics. Springer.

Flather, R.A., 1976. A tidal model of the north-west european continental shelf. Mem. Soc. R. Sci. Liege 6, 141-164.

Gustafsson, B., 2008. High order difference methods for time dependent PDE. Springer.

Gustafsson, B., Kreiss, H.O., Oliger, J., 1995. Time dependent problems and difference methods. John Wiley and Sons. Kreiss, H.O., Lorenz, J., 1989. Initial boundary value problems and the Navier-Stokes equations. Academic Press.

Mcdonald, A., 2002. A step toward transparent boundary conditions for meteorological models. Mon. Wea. Rev. 130, $140-151$.

Nordström, J., Svärd, M., 2005. Well posed boundary conditions for the Navier-Stokes equations. SIAM J. Numer. Anal. 43, 1231-1255

Oliger, J., Sundström, A., 1978. Theoretical and practical aspects of some initial boundary value problems in fluid dynamics. SIAM J. Appl. Math. 35, 419-446.

Pedlosky, J., 1987. Geophysical fluid dynamics. Springer-Verlag.

Strang, G., 1964. Accurate partial difference methods II. non-linear problems. Num. Math. 6, 37-46.

Vallis, G.K., 2006. Atmospheric and oceanic fluid dynamics: Fundamentals and large-scale circulation. Cambridge University Press. 